\title{
Evaluación de dos MÉTOdos de DIGESTIÓN ÁCIDA EN EL ANÁLISIS DE TEJIDO FOLIAR DE CAÑA (Saccharum officinarum L.)
}

\author{
EVALUATION OF TWO ACID DIGESTION METHODS IN THE ANALYSIS OF SUGAR \\ CANE LEAF TISSUE (Saccharum officinarum L.)
}

${ }^{\bullet}$ Betty Mendozal ${ }^{1}$ Lué Merú Marcó Parra ${ }^{1}$,Leyda Almao ${ }^{1}$, Vianel Rodríguez ${ }^{1}$

\begin{abstract}
${ }^{1}$ Departamento de Química y Suelos, Decanato de Agronomía. Universidad Centroccidental Lisandro Alvarado(UCLA). Barquisimeto.Venezuela.Aptdo.400.•bmendoza@ucla.edu.ve; mparra@ucla.edu.ve; leydamarina@ucla.edu.ve; vianelr@ucla.edu.ve
\end{abstract}

\section{ReSUMEN}

$\mathrm{E}^{1}$ desempeño de los métodos de digestión, vía seca (DVS) y ácida asistida por microondas (DAAM) fue evaluado en el análisis de muestras foliares de caña de azúcar (Saccharum officinarum L.). También se evaluó el efecto de las técnicas de detección comúnmente utilizadas en el laboratorio [P: espectrometría de absorción molecular visible (UV-VIS); K: espectrometría de emisión atómica (AES); $\mathrm{Ca}, \mathrm{Mg}, \mathrm{Cu}$, Zn: espectrofotometría de absorción atómica (FAAS)] respecto a la técnica de fluorescencia de rayos $\mathrm{X}$ de reflexión total (TXRF) sobre los resultados obtenidos a partir de los dos métodos de digestión. La exactitud y precisión de los métodos de digestión se determinó con dos muestras certificadas, Tejido vegetal $\mathrm{N}^{\circ} 6$ y $\mathrm{N}^{\circ} 11$ (Comisión de Normalización y Acreditación - Sociedad Chilena de la Ciencia del Suelo). La determinación de $\mathrm{P}$ vía TXRF reportó resultados mayores respecto a UV-VIS en las muestras foliares de caña de azúcar. Los métodos de digestión mostraron resultados exactos y precisos en Ca cuando se utiliza FAAS o TXRF. En Zn y $\mathrm{Cu}$ se presentan desviaciones y varianzas asociadas a las concentraciones bajas en las muestras y su relación con el blanco de reactivos. En general, la precisión y exactitud están relacionadas principalmente con el rango de concentración de cada elemento en las muestras y el límite de cuantificación. No se encontro diferencias apreciables en cuanto al desempeño analítico de ambos métodos de digestión. La detección por TXRF presenta menor eficiencia analítica en cuanto a exactitud y precisión en algunos elementos, pero menores costos en tiempo de análisis.

Palabras clave: microondas, mufla, exactitud, precisión, espectroscopia, fluorescencia.

Recibido: 27-febrero-2014. Recibido en forma corregida: 17-abril-2014.

Aceptado: 26-junio-2014.

Publicado como ARTÍCULO CIENTÍFICO en Ciencia y Tecnología 7(2): 9-20

Julio-Diciembre de 2014

ISSN 1390-4051 impreso; ISSN 1390-4043 electrónico

(C) Ciencia y Tecnología. UTEQ. Quevedo-Ecuador
Abstract

$\mathrm{T}$ he performance of via dry(DVS) and acid microwave assisted digestion methods (DAAM) was evaluated in the analysis of sugar cane leaf samples. The usual laboratory detection techniques were evaluated [P: visible molecular absorption spectrometry (UV-VIS); $\mathrm{K}$ : emission atomic spectrometry (AES); $\mathrm{Ca}, \mathrm{Mg}, \mathrm{Cu}$, $\mathrm{Zn}$ : Flame atomic absorption spectrometry] respect to the Total reflection $\mathrm{X}$ ray fluorescence technique (TXRF), for the results obtained by two digestion methods. Digestion methods accuracy and precision was determined through the use of two certified samples: plant tissue $n^{\circ} 6$ and $n^{\circ} 11$ (Accreditation and Normalization Commission - Chilean Society of Soil Science) $\mathrm{P}$ determination via TXRF reported better results with respect to UV-VIS in sugar cane leaf samples. Digestion methods showed accurate and precise results for $\mathrm{Ca}$ when using FAAS or TXRF. For $\mathrm{Zn}$ and $\mathrm{Cu}$ deviations and variances, associated to the low sample concentration and relation to reagent blank were found. In general, accuracy and precision are related mainly with the concentration range of each element in samples, and with the quantification limit. Regarding analytical performance, no significant differences were found for both digestion methods. Via TXRF detection presents lower analytical efficiency in accuracy and precision, but better performance with regard to time for analysis.

Key words: microwave, muffle, accuracy, precision, spectroscopy, fluorescence. 


\section{INTRODUCCIÓN}

$E_{\text {el }}^{1}$ análisis foliar es una herramienta esencial para diagnóstico nutricional de plantas. Consiste en medir el contenido total de los nutrientes presentes en las hojas a través de procedimientos químicos específicos. El análisis foliar se considera actualmente como una referencia indispensable para determinar tanto las necesidades de fertilización de las plantaciones como los estados carenciales de nutrientes. Esto se debe a que los análisis foliares dan una indicación precisa de la absorción de los diferentes elementos por la planta, ya que las hojas son muy sensibles a los cambios de composición del medio nutritivo (Kalra, 1998).

Para la determinación exacta de elementos nutricionales en el tejido vegetal la preparación de las muestras es una fase crítica. Generalmente una muestra sólida tiene que ser transformada en una solución para la medición del analito, lo cual es comúnmente llevado a cabo por técnicas de espectroscopia atómica: Espectrometría de absorción atómica en llama (FAAS), Espectrometría de emisión atómica (AES), Espectrometría de absorción atómica con excitación electro-térmica (ETAAS), Espectrometría de emisión óptica mediante plasma inductivamente acoplado (ICP-OES) y espectrometría de masas con plasma inductivamente acoplado (ICP-MS). La técnica de fluorescencia de rayos $\mathrm{X}$ de reflexión total (TXRF) permite la determinación simultánea de la mayoría de los analitos de interés en el análisis foliar (Martínez et al., 2008; De La Calle et al., 2013), con menores requerimientos para la preparación de las muestras.

El uso de la mayoría de estas técnicas involucra procesos de preparación de muestras para la total destrucción de la matriz por mineralización lo cual puede conducir a la pérdida del analito debido a la digestión incompleta o a la volatilización, y contaminación a partir de reactivos o cristalería del laboratorio (Wasiliewska et al., 2002; Marguí, 2006; Zudowska y Biziuk, 2008).

La digestión o mineralización de las muestras pueden realizarse por vía húmeda o digestión ácida, vía seca y en algunos casos mediante extracción por ultrasonido (Shirin et al., 2008; Untea et al., 2012). El método vía seca consiste en incinerar la muestra en el horno-mufla convencional a menos de $600{ }^{\circ} \mathrm{C}$. Altas temperaturas a veces permiten la contaminación $\mathrm{o}$ pérdida del analito y por lo tanto ha tendido a subestimar o sobrestimar. El método de digestión húmeda incluye descomposición por ácidos solos o mezclados, llevado a cabo en vasos de teflón sobre bloques de aluminio o en placas respectivamente calientes (Shirin et al., 2008), o clásicamente en vasos de precipitado sobre plancha de calentamiento.

La digestión húmeda puede realizarse en un sistema presurizado usando vasos cerrados climatizados en un horno microondas o autoclave. El uso de este sistema cerrado tiene varias ventajas con respecto a los sistemas abiertos, incluyendo la reducción significativa de los riesgos de contaminación y pérdida de analitos volátiles (Dapiaggi et al., 2001; Bocca et al., 2003). Adicionalmente, el punto de ebullición del ácido es elevado cuando la presión interna del vaso aumenta, permitiendo una más rápida y completa digestión de la matriz. Usando una mezcla de $\mathrm{HNO}_{3} \mathrm{y}_{2} \mathrm{O}_{2}$, mayor cantidad de muestra biológica puede ser completamente digerida en vasos cerrados excepto si esta tiene un alto contenido de silicatos (Hansen et al., 2009).

Sun et al. (2000), realizando comparaciones entre cinco métodos de digestión, encontraron que para los elementos evaluados el procedimiento de digestión por microondas produjo excelentes resultados, aunque este método posee una limitación relacionada al número de muestras por análisis el cual se reduce a sólo 12. El método por digestión en seco comparado con el método anterior sigue siendo favorable cuando la digestión se realiza para elementos no volátiles, ya que se pueden procesar una gran cantidad de muestras y las cenizas pueden disolverse en pequeños volúmenes.

La calidad del análisis de las plantas depende principalmente de las siguientes etapas: 1) muestreo, 2) preparación mecánica (lavado, molienda) y química de la muestra (incineración, digestión ácida), 3) análisis químico con diferentes análisis instrumentales $\mathrm{y}$ técnicas (Paslawski y Migaszewski, 2006).

En el laboratorio de la Unidad de Investigación de Suelo y Nutrición Mineral de Plantas (UISNMP) se viene realizando desde hace varios años, la digestión de las muestras foliares con fines de determinar su contenido nutricional por el método de digestión seca, el cual ha tenido inconvenientes ya que tiene una duración de 3 días por cada 40 muestras, aunque es la técnica básica de digestión o mineralización. Por otro lado, actualmente el laboratorio cuenta con el equipo digestor por microondas (ETHOS 1). Este sistema, en principio, reduce el tiempo de digestión a medio día, ofrece menor exposición del personal del laboratorio a los gases y proporciona precisión en los resultados, constituyéndose en una alternativa al método tradicionalmente usado en el laboratorio (UISNMP).

En tal sentido, considerando la necesidad de acelerar el proceso para la determinación de nutrientes, en este trabajo se compararon los métodos de digestión por vía seca y por vía húmeda asistida por microonda para así constatar sus ventajas y desventajas. También se consideró apropiado evaluar el efecto de las técnicas de detección comúnmente utilizadas en el laboratorio 
[P: espectrometría de absorción molecular visible (UVVIS); K: espectrometría de emisión atómica (AES); Ca, $\mathrm{Mg}, \mathrm{Cu}, \mathrm{Zn}$ : espectrofotometría de absorción atómica (FAAS)] y TXRF sobre los resultados obtenidos a partir de los dos métodos de digestión.

\section{Materiales y MÉTOdos}

$\mathrm{L}^{\mathrm{a}}$ a investigación se realizó bajo condiciones controladas en el laboratorio de la Unidad de Investigación de Suelo y Nutrición Mineral de Plantas (UISNMP) del Decanato de Agronomía de la UCLA, ubicado en la ciudad de Cabudare, Edo Lara.

Se analizaron diez muestras vegetales provenientes de unidades de producción agrícola, seleccionadas de acuerdo a los reportes históricos de altos rendimientos de tallos de caña de azúcar en campo, iguales o superiores a $90 \mathrm{Mg} \mathrm{ha}^{-1}$, cultivadas con la variedad de caña de azúcar Costa Rica. El área muestreada se ubica en el municipio Páez del estado Portuguesa, Venezuela. La unidad experimental está constituida por un tablón de caña, con un máximo de 10 ha, la cual fue seleccionada en cada finca, completamente al azar. Cada finca recibió dosis variables de fórmula N-P-K, sin aplicación de microelementos al suelo o a la hoja. El muestreo se realizó justo antes de la inducción floral, en el período lluvioso, y dependiendo del ciclo de cultivo, se muestrearon las unidades experimentales con 4 y 6 meses de edad, en soca y en plantilla respectivamente. Se colectaron muestras compuestas por cada unidad experimental, formada por 25 hojas con el primer labio visible llamada TVD de su abreviación del inglés Top Visible Dewlap, o "top visible dewlap" (TVD), sanas, provenientes del tallo principal o de la macolla (No TVD), como se muestra en el Cuadro 1.

Cuadro 1. Muestras foliares de caña de azúcar
variedad Costa Rica

TVD: hojas con el primer labio visible; No TDV: no hojas con el primer labio visible provenientes del tallo principal o de la macolla
De cada muestra compuesta se realizaron 4 repeticiones para cada método de digestión. Además, se analizaron 2 muestras certificadas (Tejido vegetal $\mathrm{N}^{\circ} 6 \mathrm{y}$ $\mathrm{N}^{\circ} 11$ ), provenientes de la Comisión de Normalización y Acreditación - Sociedad Chilena de la Ciencia del Suelo, con 4 repeticiones cada una, por los dos métodos de digestión. Se les determinó $\mathrm{P}, \mathrm{K}, \mathrm{Ca}, \mathrm{Mg}, \mathrm{Cu}$ y Zn.

\section{Preparación de la muestra, procedimientos y mediciones}

Acondicionamiento. Las muestras experimentales (hojas de las plantas) fueron acondicionadas en el laboratorio, de acuerdo al protocolo establecido en el manual de la FAO (2008). Éstas son lavadas con agua de chorro para la eliminación de las impurezas, luego se sumergen por 10 segundos en ácido clorhídrico al 1.2 $\mathrm{M}$ y por último en agua desionizada; se colocan a escurrir y finalmente se dejan por 48 horas en estufa con ventilación forzada a $65{ }^{\circ} \mathrm{C}$. Posteriormente, se molieron en un molino de acero inoxidable (Thomas -Willey, Philadelphia, USA) y fueron pasadas por un tamíz de acero inoxidable con malla de 20 mesh (partículas menores de $0.5 \mathrm{~mm}$ ).

Digestión por vía seca (DVS). Para la digestión por incineración de las muestras foliares de caña, se utilizó una mufla u horno (Quimis, Diadema, Brasil) con capacidad aproximada de 25 crisoles de porcelana de $30 \mathrm{~mL}$. Una masa de $500 \mathrm{mg}$ de cada muestra fue transferida a un crisol de porcelana previamente curado y tarado. Las muestras se introdujeron en la mufla y se sometieron a una rampa de calentamiento gradual, incrementando la temperatura del horno en $100{ }^{\circ} \mathrm{C}$ por cada hora hasta alcanzar los $500^{\circ} \mathrm{C}$. Una vez alcanzada la temperatura máxima, las muestras se mantuvieron en el horno durante 5 horas o hasta obtener cenizas blancas. Luego se apagó la mufla y se esperó hasta llegar a temperatura ambiente para sacar los crisoles y agregarle $5 \mathrm{~mL}$ de $\mathrm{HNO}_{3}$ concentrado por las paredes del crisol y luego $5 \mathrm{~mL}$ de agua desionizada y dejar en reposo por 14 horas. Posteriormente se trasvasó a un balón aforado de $50 \mathrm{~mL}$ con agua desionizada. Los elementos $\mathrm{Zn}$ y $\mathrm{Cu}$ se determinaron directamente en este extracto. Para $\mathrm{Ca}$ y $\mathrm{Mg}$, las muestras se diluyeron 100 veces con solución de $\mathrm{LaCl}_{3} .7 \mathrm{H}_{2} \mathrm{O}$ al $0.1 \%$, para $\mathrm{K}$, se diluyeron 25 veces también con $\mathrm{LaCl}_{3} \cdot 7 \mathrm{H}_{2} \mathrm{O}$ al $0.1 \%$. Para el $\mathrm{P}$, los extractos se diluyeron 10 veces en una solución de $\left(\mathrm{NH}_{4}\right) 6 \mathrm{Mo}_{7} \mathrm{O}_{24}+\mathrm{C}_{6} \mathrm{H}_{8} \mathrm{O}_{6}$ (molibdato de amonio + ácido ascórbico) 2:1, previa elaboración de la curva de calibración en iguales condiciones. Por cada muestra se prepararon y analizaron cuatro réplicas independientes y los respectivos blancos de reactivos. 
Digestión por microondas (DAAM). Las muestras foliares de caña fueron digeridas por vía húmeda, en el digestor de microondas (Milestone Ethos 1, Bergamo, Italy) equipado con 12 envases de $50 \mathrm{~mL}$ de capacidad, de TFM (Teflon Fluor Modified). Una masa de 500 $\mathrm{mg}$ de muestra fue transferida a los envases de teflón agregándole $7 \mathrm{~mL}$ de $\mathrm{HNO}_{3}$ concentrado $+2 \mathrm{~mL}$ de $\mathrm{H}_{2} \mathrm{O}_{2}$. En el Cuadro 2 se muestra el ciclo de tiempo, potencia y temperatura aplicada en el horno microondas para la descomposición de las muestras. Las muestras digeridas fueron transferidas a un balón de $50 \mathrm{~mL}$ con agua desionizada.

Cuadro 2. Programación del microondas para la digestión de muestras foliares

\begin{tabular}{cccc}
\hline Pasos & $\begin{array}{c}\text { Potencia } \\
\text { (watts) }\end{array}$ & $\begin{array}{c}\text { Temperatura } \\
\left({ }^{\circ} \mathbf{C}\right)\end{array}$ & $\begin{array}{c}\text { Tiempo } \\
\text { (minutos) }\end{array}$ \\
\hline 1 & 1000 & 180 & 10 \\
2 & 1000 & 180 & 15 \\
3 & 0 & 0 & 30 (enfriamiento) \\
\hline
\end{tabular}

La preparación de los extractos (diluciones y soluciones utilizadas) para las determinaciones y mediciones fue igual que para las muestras digeridas por digestión vía seca.

Agua altamente pura (conductividad eléctrica < $0.01 \mathrm{dS} \mathrm{m}^{-1}$ ) obtenida por un desionizador (Easy Pure II Ultrapure Water System, Barnstead International, Iowa, USA) con cartuchos de resinas aniónicas y catiónicas, fue utilizada para las digestiones y determinaciones en ambas metodologías. Todos los ácidos y reactivos inorgánicos usados en las digestiones, son de grado analítico: $\mathrm{HNO}_{3} \quad 62 \% \mathrm{v} / \mathrm{v}$ (LABOQG, Maracay, Venezuela) y $\mathrm{H}_{2} \mathrm{O}_{2} \quad 30 \%$ v/v (Merck, Darmstack, Alemania).

Determinación de $\mathrm{P}, \mathrm{K}, \mathrm{Ca}, \mathrm{Mg}, \mathrm{Cu}$ y $\mathrm{Zn}$ por técnicas de detección comúnmente utilizadas en el laboratorio. El espectrofotómetro de absorción atómica (Perkin Elmer 2280, Norwalk, Connecticut. USA) fue utilizado para la determinación de los siguientes elementos: $\mathrm{Ca}$, $\mathrm{Mg}, \mathrm{Cu}$ y Zn. El fotómetro de emisión de llama (Jenway PFP7, Burlington, NJ. United States) fue usado para la determinación de K. En la determinación de P se utilizó el espectrofotómetro de luz visible (Thermo Scientific Genesys 20, Verona Road, USA). Las soluciones patrones de todos los elementos, en la determinación por espectroscopia de absorción ( $\mathrm{Mg}, \mathrm{Ca}, \mathrm{Cu}$ y $\mathrm{Zn})$ o de emisión atómica $(\mathrm{K})$ fueron preparadas a partir de ampollas de 1000 mg/L (Riedel de Haen, Alemania). Para las curvas de calibración, las soluciones analíticas, se realizaron en los siguientes rangos $\left(\mathrm{mg} \mathrm{L}^{-1}\right)$; $\mathrm{Ca}: 0.5$ - 4.0; Mg: $0.2-1.0$; K: 5 - 15; Cu: $0.25-1.0$; Zn: 0.5 - 2.0. Todas las soluciones patrones para las curvas de micronutrientes, fueron diluidas en matrices de $\mathrm{HNO}$ al $9 \% \mathrm{v} / \mathrm{v}$.

Para la determinación de $\mathrm{P}$, la metodología empleada fue Olsen modificado (debido a la coloración amarilla que presentaron las muestras luego de digeridas por microondas); se utilizaron los siguientes reactivos (APHA - AWWA - WPCF, 2005): $\left(\mathrm{NH}_{4}\right)_{6} \mathrm{Mo}_{7} \mathrm{O}_{24}$ (solución ácida de molibdato de amonio) combinando con $\mathrm{C}_{8} \mathrm{H}_{4} \mathrm{~K}_{2} \mathrm{O}_{12} \mathrm{Sb}_{2} \times 3 \mathrm{H}_{2} \mathrm{O}$ (tartrato doble de potasio y antimonio), (Merck, Darmstack, Alemania) y $\mathrm{H}_{2} \mathrm{SO}_{4}$ concentrado al 96\% v/v (LABOQG, Maracay, Venezuela). La curva de calibración fue preparada por dilución de una solución de $\mathrm{KH}_{2} \mathrm{PO}_{4}$, (Merck, Darmstack, Alemania) conteniendo $1.000 \mathrm{mg} \mathrm{L}^{-1} \mathrm{de}$ fósforo, en el rango de $0.1-1 \mathrm{mg} \mathrm{L}^{-1}$.

Determinación de $\mathrm{P}, \mathrm{K}, \mathrm{Ca}, \mathrm{Mg}, \mathrm{Cu}$ y $\mathrm{Zn}$ por la técnica de fluorescencia de rayos $X$ de reflexión total (TXRF). Las determinaciones por la técnica de fluorescencia de rayos $\mathrm{X}$ de reflexión total (TXRF) se realizaron en un espectrómetro (PICOFOX, Bruker, Alemania). Todas las muestras fueron irradiadas con un ánodo de molibdeno $(17.4 \mathrm{keV})$ por un tiempo vivo de 250 segundos.

Para el análisis mediante TXRF se utilizó un método de calibración mediante estandarización interna, ampliamente descrito (Bruker, 2011a; De La Calle et al., 2013). Se utilizó una solución de cobalto de $10 \mathrm{mg} \mathrm{L}^{-1} \mathrm{de}$ concentración, preparada a partir de una solución patrón de $1000 \mathrm{mg} \mathrm{L}^{-1}$, (Riedel de Haen, Alemania). En un vial eppendorf de $1.5 \mathrm{~mL}$ de capacidad, se añadieron $500 \mu \mathrm{L}$ de muestra y $500 \mu \mathrm{L}$ de la solución de patrón interno. Posteriormente se tomaron $5 \mu \mathrm{L}$ de muestra, los cuales se depositaron sobre un portamuestras de cuarzo, el cual es irradiado posteriormente, como se describe en la sección de instrumentos y equipos. La cuantificación de los elementos se realiza de forma simultánea mediante el programa SPECTRA de PICOFOX (Bruker, 2011b).

Tiempo de ejecución de los métodos. Para ambos métodos de digestión se midieron los tiempos de ejecución, comenzando desde la preparación del material, pesaje, digestión, trasvasado y obtención del extracto final. Adicionalmente, se consideró el tiempo para las técnicas de detección.

Análisis estadísticos. La base de datos se elaboró con las concentraciones de $\mathrm{P}, \mathrm{K}, \mathrm{Ca}, \mathrm{Mg}, \mathrm{Cu}$ y $\mathrm{Zn}$ en diez muestras foliares detallados en el Cuadro 1. El modelo estadístico aplicado se corresponde con un experimento factorial con dos factores: método de digestión (DVS y DAAM) y técnica de detección (comúnmente utilizadas en el laboratorio: P por UV-VIS; K por AES y Ca, Mg, $\mathrm{Cu}, \mathrm{Zn}$ por FAAS; método alternativo: TXRF). Para 
cada combinación de método de digestión y técnica de detección de las 10 muestras se hicieron cuatro repeticiones. El modelo estadístico correspondiente es:

$y_{i j k}=\mu+F_{i}+P_{j}+(F P)_{i j}+\varepsilon_{i j k}$

Donde $\mu$ es la media, $F_{i}$ es el efecto debido al método de digestión, $P_{j}$ es el efecto debido a la técnica de detección, $(F P)_{i j}$ es el efecto de la interacción entre el método de digestión y la técnica de detección, y $\varepsilon_{i j k}$ es el error.

En los casos que fue necesario se realizaron comparaciones de medias por la prueba de la diferencia honestamente significativa (DHS) de Tukey $(\mathrm{p}<0.05)$.

Para evaluar la exactitud y precisión de los métodos de digestión con las dos muestras de referencia mencionadas al inicio de la sección, Tejido vegetal $\mathrm{N}^{\circ} 6$ y $\mathrm{N}^{\circ} 11$, los resultados obtenidos mediante los dos métodos de detección, por separado, fueron analizados estadísticamente mediante análisis de varianza. La comparación de medias de cada tratamiento (DVS y DAAM) con respecto al valor real se hizo a través de la prueba de hipótesis individual ( $t$ de student). Para estimar la precisión, a las concentraciones de cada elemento se determinó el valor medio, desviación estándar y su coeficiente de variación (CV).

Todas las inferencias estadísticas fueron hechas a un nivel de probabilidad de 0.05 . Los datos fueron analizados utilizando el programa estadístico INFOSTAT (2002). Así mismo se hizo análisis comparativo de las ventajas y desventajas de cada uno de los métodos con base en los resultados obtenidos.

\section{ReSUltados Y Discusión}

\section{Muestras problema}

Para el fósforo se observa que no hay diferencias significativas entre ambos métodos de digestión para tres $(804,806,808)$ de las diez muestras, independientemente del método de detección que se utilice (Cuadro 3). Hay diferencias significativas entre ambos métodos de digestión para cinco de las muestras $(805,807,590,792,798)$ cuando se utiliza la detección colorimétrica, esto puede derivarse de la diferencia en coloración que presentan las muestras por ambos métodos de digestión, las cuales son incoloras cuando se realiza la digestión vía seca. Aunque el método de detección por TXRF muestra menos discrepancias entre ambos métodos de digestión, tres de las diez muestras son diferentes $(809,588,590)$; se observan diferencias significativas respecto al método de detección colorimétrico en la mayor parte de las muestras, siendo los valores siempre significativamente mayores por
TXRF. Esto se debe a que el elemento tiene una línea analítica por rayos $\mathrm{X}$ en la cual se presentan importantes interferencias espectrales y de matriz, por parte de otros elementos presentes en las muestras foliares de caña de azúcar, y posee una línea de fondo que no puede ser ajustada adecuadamente, pudiendo resultar una sobreestimación cuando la concentración del elemento en el spot analizado es del orden de $10 \mathrm{mg} \mathrm{L}^{-1}$.

Para el potasio se observan valores significativamente mayores por el método del microondas en cuatro de las muestras $(807,588,590$, 792) independientemente del método de detección utilizado, para tres de las muestras $(806,808,809)$ con detección por espectrometría atómica (Cuadro 3). Esto se debe a pérdidas durante el procedimiento de DVS (Sun et al., 2000).

En cuatro de las muestras $(805,806,807,798)$ no se observan diferencias estadísticas debido al método de detección, independientemente del método de digestión aplicado. Por otro lado, en las muestras 588, 590 y 792 se encontró, que independientemente del método de digestión aplicado, los valores de concentración de potasio por AES fueron significativamente superiores a los obtenidos por TXRF (Cuadro 3).

Los resultados para el calcio presentados en el Cuadro 3, en general, no muestran diferencias estadísticas atribuidas al método de digestión utilizado (804, 805, 806, 807, 809, 798), así como tampoco respecto al método de detección $(804,805,806,807$, $808,809,588,798)$. En relación con el magnesio, no se observan diferencias en los resultados atribuibles al método de digestión $(804,805,806,808,809,588,590$, 792). El procedimiento de detección mediante absorción atómica en llama presentó coeficientes de varianza bajos. En los casos en los cuales fue posible la detección mediante TXRF, tampoco se observaron diferencias derivadas del método de digestión o de detección, aunque se observaron varianzas significativamente altas por el método TXRF (805, 808, 588, 590). Es importante acotar que este es un elemento ligero de difícil determinación por la técnica de rayos $\mathrm{X}$, lo cual demanda una optimización que escapa a los objetivos del presente estudio. En este caso sería conveniente una dilución menor de la muestra, debido a que el límite de detección en el spot analizado fue de $4 \mathrm{mg} \mathrm{L}^{-1}$ por el método de vía seca y de $5 \mathrm{mg} \mathrm{L}^{-1}$ por el método de vía húmeda. Para una concentración del orden de $0.10 \%$ en las muestras secas, la dilución empleada en los procedimientos de digestión (cien veces), llevó al analito al límite de cuantificación.

Los elementos que presentaron mayor concordancia con ambos métodos de mineralización y con los dos métodos de detección empleados fueron el magnesio, el calcio y el potasio (Cuadro 3). Los coeficientes de 
Cuadro 3. Concentraciones de $\mathrm{P}, \mathrm{K}, \mathrm{Ca}$ y Mg en muestras foliares de caña de azúcar determinada por dos métodos de digestión

\begin{tabular}{|c|c|c|c|c|c|c|c|c|c|}
\hline \multirow[b]{2}{*}{ Muestra } & \multirow[b]{2}{*}{ Método } & \multicolumn{2}{|c|}{$\mathbf{P}(\%)$} & \multicolumn{2}{|c|}{ K (\%) } & \multicolumn{2}{|c|}{ Ca (\%) } & \multicolumn{2}{|c|}{$\operatorname{Mg}(\%)$} \\
\hline & & UV-VIS & TXRF & AES & TXRF & FAAS & TXRF & FAAS & TXRF \\
\hline \multirow{4}{*}{804} & DAAM & $0.19 \pm 0.01$ & $0.26 \pm 0.03$ & $1.58 \pm 0.01$ & $1.9 \pm 0.3$ & $0.32 \pm 0.04$ & $0.31 \pm 0.05$ & $0.10 \pm 0.01$ & $\overline{\mathrm{ND}}$ \\
\hline & & $(5.0 \%) b$ & $(12 \%) \mathrm{a}$ & $(0.6 \%) b$ & $(16 \%) a$ & $(13.4 \%) \mathrm{a}$ & $(16 \%) \mathrm{a}$ & $(6.1 \%) b$ & \\
\hline & DVS & $0.18 \pm 0.01$ & $0.25 \pm 0.02$ & $1.58 \pm 0.02$ & $1.7 \pm 0.1$ & $0.32 \pm 0.06$ & $0.26 \pm 0.03$ & $0.09 \pm 0.00$ & $0.117 \pm 0.00$ \\
\hline & & $(2.8 \%) b$ & $(8 \%) a$ & $(1.2 \%) b$ & $(6 \%) \mathrm{ab}$ & $(17.79 \%) \mathrm{a}$ & $(11.5 \%) \mathrm{a}$ & $(0.0 \%) b$ & $(3.4 \%) \mathrm{a}$ \\
\hline \multirow{4}{*}{805} & DAAM & $0.20 \pm 0.00$ & $0.25 \pm 0.03$ & $1.44 \pm 0.03$ & $1.4 \pm 0.1$ & $0.51 \pm 0.05$ & $0.45 \pm 0.05$ & $0.11 \pm 0.01$ & $0.10 \pm 0.05$ \\
\hline & & $(0.0 \%) b$ & $(12 \%) \mathrm{a}$ & $(2.3 \%) a$ & $(7 \%) \mathrm{a}$ & $(10.2 \%) \mathrm{a}$ & $(11 \%) \mathrm{a}$ & $(4.4 \%) a$ & $(50 \%) \mathrm{a}$ \\
\hline & DVS & $0.18 \pm 0.00$ & $0.27 \pm 0.02$ & $1.41 \pm 0.02$ & $1.7 \pm 0.3$ & $0.51 \pm 0.07$ & $0.5 \pm 0.1$ & $0.11 \pm 0.01$ & $0.15 \pm 0.04$ \\
\hline & & $(0.0 \%) \mathrm{c}$ & $(7 \%) a$ & $(1.42 \%) \mathrm{a}$ & $(18 \%) \mathrm{a}$ & $(13.1 \%) \mathrm{a}$ & $(20 \%) a$ & $(4.6 \%) \mathrm{a}$ & $(27 \%) \mathrm{a}$ \\
\hline \multirow{4}{*}{806} & DAAM & $0.22 \pm 0.01$ & $0.30 \pm 0.02$ & $2.04 \pm 0.02$ & $2.10 \pm 0.06$ & $0.33 \pm 0.04$ & $0.31 \pm 0.05$ & $0.10 \pm 0.01$ & ND \\
\hline & & $(4.3 \%) b$ & $(7 \%) a$ & $(1.2 \%) \mathrm{a}$ & $(3 \%) a$ & $(12.12 \%) \mathrm{a}$ & $(16 \%) \mathrm{a}$ & $(5.1 \%) \mathrm{a}$ & \\
\hline & DVS & $0.20 \pm 0.01$ & $0.30 \pm 0.05$ & $1.88 \pm 0.02$ & $1.9 \pm 0.3$ & $0.32 \pm 0.06$ & $0.26 \pm 0.09$ & $0.10 \pm 0.01$ & ND \\
\hline & & $(6.4 \%) b$ & $(17 \%) a$ & $(0.92 \%) b$ & $(16 \%) a b$ & $(18.1 \%) \mathrm{a}$ & $(35 \%) \mathrm{a}$ & $(5.1 \%) \mathrm{a}$ & \\
\hline \multirow{4}{*}{807} & DAAM & $0.20 \pm 0.01$ & $0.27 \pm 0.02$ & $2.19 \pm 0.02$ & $2.4 \pm 0.2$ & $0.26 \pm 0.03$ & $0.28 \pm 0.08$ & $0.090 \pm 0.00$ & $0.14 \pm 0.02$ \\
\hline & & $(4.1 \%) b$ & $(7 \%) a$ & $(0.7 \%) \mathrm{a}$ & $(8 \%) a$ & $(12.97 \%) \mathrm{a}$ & $(29 \%) a$ & $(0.0 \%) \mathrm{b}$ & $(14 \%) \mathrm{a}$ \\
\hline & DVS & $0.17 \pm 0.00$ & $0.25 \pm 0.01$ & $1.96 \pm 0.04$ & $1.9 \pm 0.2$ & $0.28 \pm 0.06$ & $0.26 \pm 0.01$ & $0.092 \pm 0.00$ & ND \\
\hline & & $(0.0 \%) \mathrm{c}$ & $(4 \%) a$ & $(1.8 \%) b$ & $(10.5 \%) b$ & $(19.5 \%) \mathrm{a}$ & $(4 \%) a$ & $(5.4 \%) \mathrm{a}$ & \\
\hline \multirow{4}{*}{808} & DAAM & $0.21 \pm 0.01$ & $0.30 \pm 0.02$ & $2.14 \pm 0.05$ & $1.9 \pm 0.15$ & $0.29 \pm 0.04$ & $0.29 \pm 0.08$ & $0.080 \pm 0.00$ & $0.10 \pm 0.05$ \\
\hline & & $(4.5 \%) b$ & $(7 \%) a$ & $(2.3 \%) \mathrm{a}$ & $(8 \%) b$ & $(15.56 \%) a$ & $(28 \%) \mathrm{a}$ & $(0.0 \%) \mathrm{a}$ & $(50 \%) \mathrm{a}$ \\
\hline & DVS & $0.20 \pm 0.01$ & $0.30 \pm 0.02$ & $1.69 \pm 0.06$ & $1.9 \pm 0.1$ & $0.20 \pm 0.02$ & $0.21 \pm 0.08$ & $0.080 \pm 0.00$ & ND \\
\hline & & $(2.5 \%) b$ & $(7 \%) a$ & $(3.9 \%) \mathrm{c}$ & $(5 \%) \mathrm{b}$ & $(8.2 \%) b$ & $(38 \%) b$ & $(0.0 \%) \mathrm{a}$ & \\
\hline \multirow{4}{*}{809} & DAAM & $0.24 \pm 0.01$ & $0.30 \pm 0.01$ & $2.26 \pm 0.03$ & $2.0 \pm 0.2$ & $0.26 \pm 0.01$ & $0.27 \pm 0.03$ & $0.090 \pm 0.00$ & ND \\
\hline & & $(3.4 \%) \mathrm{c}$ & $(3.3 \%) b$ & $(1.5 \%) \mathrm{ab}$ & $(10 \%) b$ & $(3.8 \%) \mathrm{a}$ & $(11 \%) \mathrm{a}$ & $(0.0 \%) \mathrm{a}$ & \\
\hline & DVS & $0.22 \pm 0.01$ & $0.35 \pm 0.02$ & $1.84 \pm 0.06$ & $2.3 \pm 0.4$ & $0.27 \pm 0.02$ & $0.29 \pm 0.07$ & $0.090 \pm 0.00$ & ND \\
\hline & & $(4.6 \%) c$ & $(6 \%) a$ & $(3.1 \%) c$ & $(17 \%) \mathrm{a}$ & $(6.0 \%) \mathrm{a}$ & $(24 \%) a$ & $(0.0 \%) \mathrm{a}$ & \\
\hline \multirow{4}{*}{588} & DAAM & $0.18 \pm 0.02$ & $0.24 \pm 0.01$ & $1.69 \pm 0.02$ & $1.50 \pm 0.06$ & $0.41 \pm 0.04$ & $0.49 \pm 0.05$ & $0.14 \pm 0.00$ & $0.15 \pm 0.04$ \\
\hline & & $(9.6 \%) b$ & $(4 \%) a$ & $(1.1 \%) \mathrm{a}$ & $(4 \%) b$ & $(9.4 \%) b$ & $(10 \%) a b$ & $(0.0 \%) a$ & $(27 \%) \mathrm{a}$ \\
\hline & DVS & $0.15 \pm 0.01$ & $0.14 \pm 0.02$ & $1.42 \pm 0.02$ & $1.2 \pm 0.1$ & $0.52 \pm 0.05$ & $0.46 \pm 0.05$ & $0.15 \pm 0.01$ & $0.13 \pm 0.02$ \\
\hline & & $(5.44) \mathrm{b}$ & $(14 \%) b$ & $(1.5 \%) b$ & $(8 \%) c$ & $(9.0 \%) \mathrm{a}$ & $(11 \%) a b$ & $(6.9 \%) a$ & $(15 \%) \mathrm{a}$ \\
\hline \multirow{4}{*}{590} & DAAM & $0.18 \pm 0.01$ & $0.23 \pm 0.01$ & $1.64 \pm 0.01$ & $1.4 \pm 0.1$ & $0.46 \pm 0.02$ & $0.48 \pm 0.03$ & $0.15 \pm 0.01$ & $0.14 \pm 0.04$ \\
\hline & & $(5.4 \%) \mathrm{b}$ & $(4 \%) a$ & $(0.5 \%) \mathrm{a}$ & $(7 \%) b$ & $(3.5 \%) \mathrm{c}$ & $(6 \%) c$ & $(3.3 \%) \mathrm{a}$ & $(29 \%) \mathrm{a}$ \\
\hline & DVS & $0.15 \pm 0.01$ & $0.16 \pm 0.08$ & $1.40 \pm 0.04$ & $1.26 \pm 0.03$ & $0.53 \pm 0.10$ & $0.517 \pm 0.002$ & $0.15 \pm 0.02$ & $0.14 \pm 0.4$ \\
\hline & & $(3.3 \%) \mathrm{c}$ & $(50 \%) \mathrm{bc}$ & $(2.6 \%) b$ & $(2 \%) c$ & $(19 \%) \mathrm{a}$ & $(0.4 \%) b$ & $(12.8 \%) \mathrm{a}$ & $(29 \%) \mathrm{a}$ \\
\hline \multirow{4}{*}{792} & DAAM & $0.25 \pm 0.01$ & $0.326 \pm 0.009$ & $1.80 \pm 0.02$ & $1.6 \pm 0.1$ & $0.50 \pm 0.02$ & $0.57 \pm 0.03$ & $0.14 \pm 0.01$ & ND \\
\hline & & $(3.9 \%) b$ & $(3 \%) a$ & $(1.0 \%) \mathrm{a}$ & $(6 \%) b$ & $(4.1 \%) b$ & $(4 \%) a$ & $(3.6 \%) \mathrm{a}$ & \\
\hline & DVS & $0.22 \pm 0.01$ & $0.31 \pm 0.03$ & $1.45 \pm 0.03$ & $1.32 \pm 0.07$ & $0.54 \pm 0.03$ & $0.47 \pm 0.01$ & $0.14 \pm 0.00$ & ND \\
\hline & & $(6.0 \%) \mathrm{c}$ & $(10 \%) \mathrm{a}$ & $(2.0 \%) \mathrm{c}$ & $(5 \%) d$ & $(5.5 \%) \mathrm{ab}$ & $(2 \%) b$ & $(0.0 \%) \mathrm{a}$ & \\
\hline \multirow{4}{*}{798} & DAAM & $0.25 \pm 0.01$ & $0.32 \pm 0.02$ & $1.67 \pm 0.10$ & $1.7 \pm 0.1$ & $0.48 \pm 0.04$ & $0.55 \pm 0.05$ & $0.12 \pm 0.00$ & ND \\
\hline & & $(2.0 \%) \mathrm{b}$ & $(6 \%) a$ & $(6.0 \%) \mathrm{ab}$ & $(6 \%) \mathrm{a}$ & $(8.09 \%) \mathrm{a}$ & $(9 \%) a$ & $(0.0 \%) \mathrm{a}$ & \\
\hline & DVS & $0.20 \pm 0.00$ & $0.31 \pm 0.03$ & $1.55 \pm 0.02$ & $1.6 \pm 0.3$ & $0.50 \pm 0.02$ & $0.49 \pm 0.02$ & $0.11 \pm 0.00$ & $0.12 \pm 0.04$ \\
\hline & & $(0.0 \%) \mathrm{c}$ & $(10 \%) \mathrm{a}$ & $(1.0 \%) b$ & $(19 \%) a b$ & $(4.8 \%) \mathrm{a}$ & $(4 \%) a$ & $(0.0 \%) b$ & $(33 \%) \mathrm{a}$ \\
\hline
\end{tabular}

DAAM= digestión ácida asistida por microondas; DVS= digestión vía seca; UV-VIS= espectrometría de absorción molecular visible; $(\mathrm{AES})=$ espectrometría de emisión atómica; $(\mathrm{FAAS})=$ espectrofotometría de absorción atómica; TXRF= técnica de fluorescencia de rayos X de reflexión total; $\mathrm{ND}=$ no detectable. Los valores corresponden al promedio \pm desviación estándar (4 réplicas). El valor entre paréntesis indica la desviación estándar relativa de las medidas Medias seguidas por la misma letra no presentan diferencias estadísticas (Tukey, $\mathrm{p}<0.05$ ). 
varianza de calcio fueron significativamente mayores a los obtenidos para potasio y magnesio con ambos métodos de digestión, y detección por espectrometría atómica, lo que indica una distribución menos homogénea de este elemento en las muestras problema. El coeficiente de varianza para el potasio varió entre el 1-20\%, es menor al reportado por Martínez et al. (2008), cuando determinaron el elemento en las muestras certificadas (NIST 1572 Citrus leaves y NIST 1573 Tomato leaves) por la técnica TXRF anteriormente digeridas por el método de digestión húmeda asistida por microonda.

El contenido de $\mathrm{Zn}$ en cinco (808, 809, 588, 590, 798) de las diez muestras de tejido foliar de caña de azúcar no mostró diferencias significativas derivadas del método de digestión independientemente del método de detección aplicado (Cuadro 4). Del resto de las muestras, cuatro $(804,805,806,792)$ sólo mostraron diferencia atribuida al método digestión cuando la detección se hizo por absorción atómica en llama. La concentración fue mayor cuando se utilizó DAAM, esto debido a que este procedimiento involucra mayor cantidad de reactivos y posibilidad de blancos más altos.

Los resultados de $\mathrm{Zn}$ en general, muestran que TXRF da valores significativamente superiores al método de detección FAAS (Cuadro 4). Cuando se utiliza la detección con TXRF, se observa que a las concentraciones estudiadas (del orden de $20 \mathrm{mg}$ $\mathrm{kg}^{-1}$ ), los spots analizados tienen concentraciones del elemento del orden de $0.10-0.20 \mathrm{mg} \mathrm{L}^{-1}$, mientras que el blanco de reactivos, sumado a la contribución del blanco del patrón interno añadido, se encuentra en el orden de $0.040 \mathrm{mg} \mathrm{L}^{-1}$. Este hecho es determinante en el desempeño poco eficiente de la técnica TXRF para la determinación del elemento a los niveles presentes en el tejido foliar evaluado. Se requeriría un método de digestión con una dilución menor o con mayor masa de muestra.

En cinco muestras $(804,805,806,590,792)$ no se observó diferencias en el contenido de $\mathrm{Cu}$ por efecto del método de digestión cuando se trabajó con el método de detección FAAS (Cuadro 4). Sin embargo, en estas mismas muestras, cuando se utilizó TXRF los valores de $\mathrm{Cu}$ en DVS fueron significativamente superiores a los de DAAM. Así mismo, las muestras 807, 809, 588 y 590 también muestran valores significativamente mayores con DAAM en detección por espectrometría de absorción atómica.

El comportamiento del $\mathrm{Cu}$ ante los diferentes métodos de digestión es variable y se relaciona con la concentración de este analito en las muestras, que, por un lado es la menor entre todos los elementos cuantificados y por otro lado, se encuentra en el límite de cuantificación de la técnica de absorción atómica en
Cuadro 4. Concentraciones de $\mathrm{Zn}$ y $\mathrm{Cu}$ en muestras foliares de caña de azúcar determinada por dos métodos de digestión

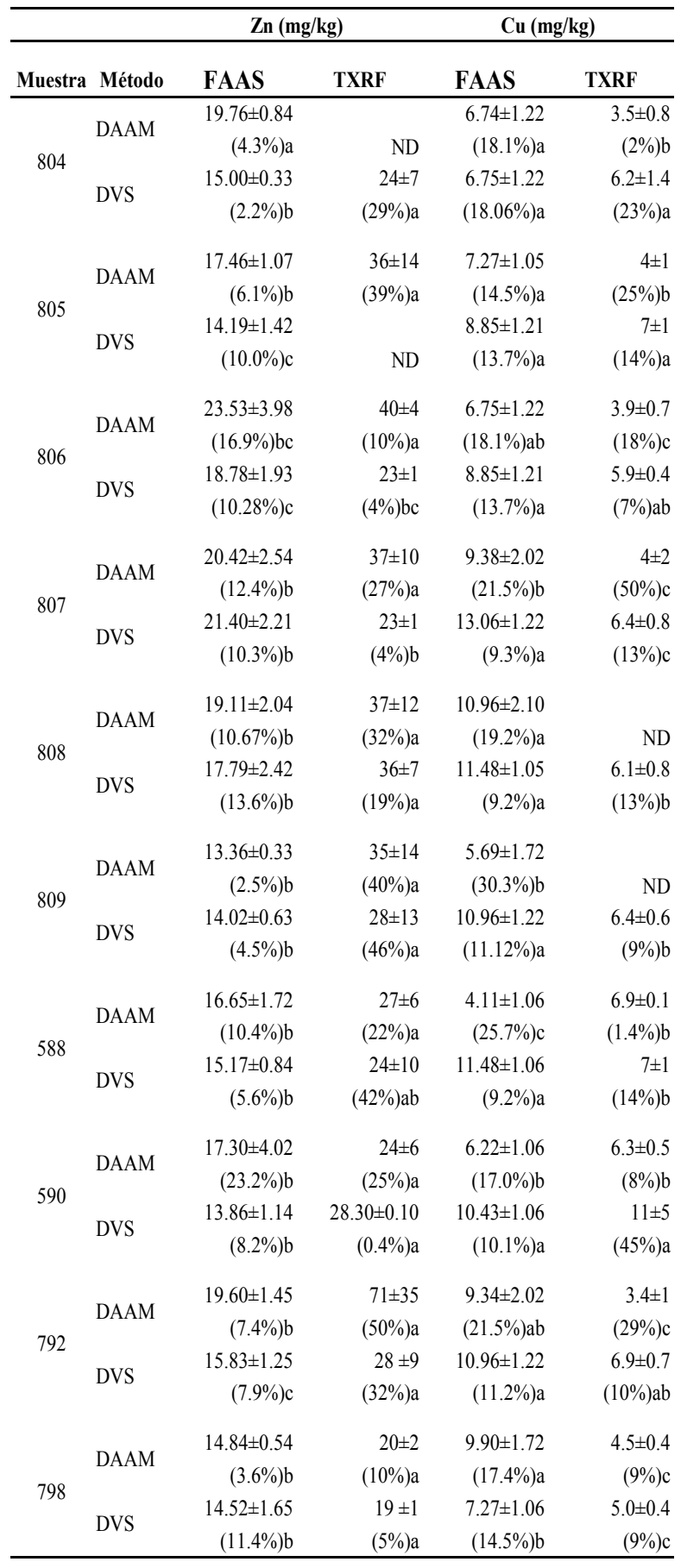

$\mathrm{DAAM}=$ digestión ácida asistida por microondas; DVS= digestión vía seca; (FAAS) = espectrofotometría de absorción atómica; TXRF= técnica de fluorescencia de rayos $\mathrm{X}$ de reflexión total; $\mathrm{ND}=$ no detectable. Los valores corresponden al promedio \pm desviación estándar (4 réplicas). El valor entre paréntesis indica la desviación estándar relativa de las medidas.

Medias seguidas por la misma letra no presentan diferencias estadísticas (Tukey, $\mathrm{p}<0.05$ ). 
llama, de $5 \mathrm{mg} \mathrm{kg}^{-1}$ (Cuadro 4).

En líneas generales, los resultados son menores con TXRF respecto a FAAS, cuando se presentan diferencias significativas entre los dos métodos de detección (Cuadro 4). En las muestras de campo (Cuadro 3 y 4) el nutriente que presentó menor variación entre réplicas fue el potasio, con $\mathrm{CV}$ entre 0.5 y $6 \%$, seguido de fósforo y magnesio. Mientras que el cobre revela menor precisión en su determinación puesto que presenta los $\mathrm{CV}$ más altos, variando entre 9.2 y $30.3 \%$, se aprecia claramente que el método de DAAM es menos preciso que DVS, no así en el resto de los elementos determinados.

La evaluación de los resultados para 10 muestras problema de tejido foliar de caña, permite inferir que la incidencia del procedimiento de digestión aplicado depende de cada elemento determinado, su rango de concentración en las muestras y la homogeneidad de su distribución. Por otra parte, el método seleccionado para la mineralización de las muestras debe ajustarse a los requerimientos de la técnica de detección en cada caso, siendo necesario tener en cuenta que no se produzca una dilución del analito que afecte la calidad analítica de los resultados en términos de precisión y exactitud, como tampoco interferencias espectrales o de matriz.

\section{Exactitud y precisión de los dos métodos de digestión con muestras de referencia}

Detección mediante espectrometría atómica (Mg, $\mathbf{K}, \mathbf{C a}, \mathbf{C u}$ y Zn) y colorimetría (P). En la muestra certificada $\mathrm{N}^{\circ} 6$ la concentración del elemento P mostró diferencias significativas entre el valor real y los valores obtenidos a partir de los dos métodos de digestión evaluados, siendo significativamente inferior al valor real (Cuadro 5). Sin embargo, en la muestra certificada $\mathrm{N}^{\circ} 11$ no se observan diferencias significativas entre los valores obtenidos por los dos métodos de digestión y el valor real (Cuadro 6). Se observan varianzas bajas para la muestra $N^{o} 11$, mientras que para la muestra 6 la varianza fue mayor al $10 \%$ por el método de DAAM. Todo indica que dependiendo de la matriz y el rango de concentración, ambos métodos pueden llegar a ser exactos y precisos para el análisis de este elemento. En la muestra certificada $\mathrm{N}^{\circ} 6$ el Error (Er) en la DAAM fue el mayor (21\%) por lo que el valor obtenido con este método se aleja más del valor real, lo cual refleja la influencia del color de los extractos en el rango bajo de concentración del elemento en las muestras (menor a $0.10 \%$ ). En la digestión vía seca todas las muestras resultan incoloras y no se presenta este factor.

Cuadro 5. Concentración de $\mathrm{P}, \mathrm{K}, \mathrm{Ca}, \mathrm{Mg}, \mathrm{Zn}$ y $\mathrm{Cu}$ en la muestra vegetal certificada $N^{\circ} 6$ (CNA-Chile) determinada por dos métodos de digestión. Detección mediante espectrometría atómica $(\mathrm{Mg}, \mathrm{K}, \mathrm{Ca}, \mathrm{Cu}$ y $\mathrm{Zn})$ y colorimetría $(\mathrm{P})$

\begin{tabular}{lcrrrrrrr}
\hline \multirow{2}{*}{ Elemento } & $\begin{array}{c}\text { VR } \\
\boldsymbol{\%}\end{array}$ & $\begin{array}{c}\text { CV } \\
\boldsymbol{\%}\end{array}$ & $\begin{array}{c}\text { DAAM } \\
\boldsymbol{\%}\end{array}$ & $\begin{array}{c}\text { Er DAAM } \\
\boldsymbol{\%}\end{array}$ & $\begin{array}{c}\text { CV } \\
\boldsymbol{\%}\end{array}$ & $\begin{array}{c}\text { DVS } \\
\boldsymbol{\%}\end{array}$ & $\begin{array}{c}\text { Er DVS } \\
\boldsymbol{\%}\end{array}$ & $\begin{array}{c}\text { CV } \\
\boldsymbol{\%}\end{array}$ \\
\hline $\mathrm{P}$ & $0.086 \mathrm{aA}$ & 10 & $0.068 \mathrm{~b}$ & 21 & 14.18 & $0.078 \mathrm{~B}$ & 9 & 6.45 \\
$\mathrm{~K}$ & $0.640 \mathrm{aA}$ & 9 & $0.640 \mathrm{a}$ & 0 & 2.73 & $0.640 \mathrm{~A}$ & 0 & 4.32 \\
$\mathrm{Ca}$ & $1.250 \mathrm{aA}$ & 7 & $1.320 \mathrm{a}$ & 6 & 5.56 & $1.280 \mathrm{~A}$ & 2 & 9.17 \\
$\mathrm{Mg}$ & $0.175 \mathrm{aB}$ & 8 & $0.190 \mathrm{a}$ & 9 & 2.67 & $0.200 \mathrm{~A}$ & 14 & 2.96 \\
$\mathrm{Zn}^{*}$ & $19.000 \mathrm{aA}$ & 10 & $21.170 \mathrm{a}$ & 11 & 15.59 & $15.120 \mathrm{~B}$ & 20 & 5.14 \\
$\mathrm{Cu}^{*}$ & $5.100 \mathrm{aB}$ & 22 & $6.220 \mathrm{a}$ & 22 & 32.49 & $9.380 \mathrm{~A}$ & 84 & 11.20 \\
\hline
\end{tabular}

$\mathrm{VR}=$ valor real; $\mathrm{DAAM}=$ digestión ácida asistida por microondas; $\mathrm{DVS}=$ digestión vía seca; $\mathrm{Er}=$ error relativo porcentual. * Concentración en $\mathrm{mg} \mathrm{kg}^{-1}$.

Medias seguidas por la misma letra minúscula en la fila para VR y DAAM y mayúscula para VR y DVS no presentan diferencias estadísticas (Tukey, $\mathrm{p}<0.05$ ).

En la muestra $\mathrm{N}^{\mathrm{o}} 6$ ambos métodos fueron exactos y precisos en la determinación de $\mathrm{K}$, mientras que en la 11 sólo fue exacto el método de DVS, aunque con el método de digestión DAAM el error no fue considerablemente apreciable (11\%).

El Ca no mostró diferencia significativa entre las concentraciones obtenidas con los dos métodos evaluados con respecto al valor real, revelando que ambos métodos son exactos para la determinación de estos elementos, lo cual es corroborado con los valores de Er entre 2-6\% para ambas muestras certificadas. La precisión para este elemento en ambos métodos es mayor que para los elementos $\mathrm{P}, \mathrm{Mg}$ y $\mathrm{K}$.

En las muestras de caña de azúcar el magnesio no mostró diferencias atribuidas al método de digestión, en el caso de las muestras certificadas sigue un comportamiento similar, aunque, la concentración de Mg obtenida a partir de la DAAM fue estadísticamente igual a la real, no así para la DVS que arrojó un valor significativamente superior al real. Sin embargo, 
Cuadro 6. Concentración de $\mathrm{P}, \mathrm{K}, \mathrm{Ca}, \mathrm{Mg}, \mathrm{Zn}$ y $\mathrm{Cu}$ en la muestra vegetal certificada $N^{\circ} 11$ (CNA-Chile) determinada por dos métodos de digestión. Detección mediante espectrometría atómica $(\mathrm{Mg}, \mathrm{K}, \mathrm{Ca}, \mathrm{Cu}$ y $\mathrm{Zn})$ y colorimetría (P)

\begin{tabular}{|c|c|c|c|c|c|c|c|c|}
\hline Elemento & $\begin{array}{l}\text { VR } \\
\%\end{array}$ & $\begin{array}{l}\mathrm{CV} \\
\%\end{array}$ & $\begin{array}{c}\text { DAAM } \\
\%\end{array}$ & $\begin{array}{c}\text { Er DAAM } \\
\%\end{array}$ & $\begin{array}{c}\mathrm{CV} \\
\%\end{array}$ & $\begin{array}{c}\text { DVS } \\
\%\end{array}$ & $\begin{array}{c}\text { Er DVS } \\
\%\end{array}$ & $\begin{array}{c}\mathrm{CV} \\
\%\end{array}$ \\
\hline $\mathrm{P}$ & $0.229 \mathrm{aA}$ & 5 & $0.22 \mathrm{a}$ & 4 & 4.40 & $0.22 \mathrm{~A}$ & 4 & 2.30 \\
\hline K & $1.640 \mathrm{bA}$ & 6 & $1.82 \mathrm{a}$ & 11 & 2.01 & $1.60 \mathrm{~A}$ & 2 & 1.56 \\
\hline $\mathrm{Ca}$ & $2.070 \mathrm{aA}$ & 4 & $2.12 \mathrm{a}$ & 2 & 6.43 & $2.01 \mathrm{~A}$ & 3 & 2.47 \\
\hline $\mathrm{Mg}$ & $0.380 \mathrm{aB}$ & 5 & $0.39 \mathrm{a}$ & 3 & 1.27 & $0.40 \mathrm{~A}$ & 5 & 3.19 \\
\hline $\mathrm{Zn} *$ & $58.00 \mathrm{aA}$ & 4 & $55.98 \mathrm{~b}$ & 3 & 0.94 & $56.94 \mathrm{~A}$ & 2 & 1.76 \\
\hline $\mathrm{Cu}^{*}$ & $19.00 \mathrm{aB}$ & 10 & $19.92 \mathrm{a}$ & 5 & 5.30 & $24.14 \mathrm{~A}$ & 26 & 8.37 \\
\hline
\end{tabular}

$\mathrm{VR}=$ valor real; $\mathrm{DAAM}=$ digestión ácida asistida por microondas; $\mathrm{DVS}=$ digestión vía seca; $\mathrm{Er}=$ error relativo porcentual. * Concentración en $\mathrm{mg} \mathrm{kg}^{-1}$.

Medias seguidas por la misma letra minúscula en la fila para VR y DAAM y mayúscula para VR y DVS no presentan diferencias estadísticas (Tukey, $\mathrm{p}<0.05$ ).

las desviaciones respecto al valor certificado no superan el 5\% para la muestra de referencia de mayor concentración.

El método de DAAM fue exacto para la determinación de $\mathrm{Zn}$ en la muestra certificada $\mathrm{N}^{\circ} 6$, no así la DVS que reveló un valor significativamente inferior; lo contario se encontró en la muestra $\mathrm{N}^{\circ} 11$.

En ambas muestras certificadas la DAAM fue exacta en la determinación de $\mathrm{Cu}$, obteniéndose valores significativamente superiores al real con la DVS.

En la muestra $\mathrm{N}^{\circ} 6$ los elementos que presentaron mayor precisión en su determinación fueron $\mathrm{K}$ y $\mathrm{Mg}$, con $\mathrm{CV}$ por debajo de $5 \%$ con ambos métodos, mientras que $\mathrm{Cu}, \mathrm{Zn}$ y $\mathrm{P}$ dieron resultados menos precisos y más aun a partir de DAAM.

La muestra certificada $\mathrm{N}^{\circ} 11$ deja ver mayor precisión en los resultados obtenidos, puesto que sólo en el $\mathrm{Cu}$ y $\mathrm{Ca}$ se encontraron valores de $\mathrm{CV}$ mayores a $5 \%$ siendo el máximo de $8.37 \%$ en Cu por DVS. La precisión se relaciona con el rango de concentración de los elementos en las muestras, que es más alto en la muestra $\mathrm{N}^{\circ} 11$ y por otro lado con los diferentes procesos ejecutados en la preparación (Montero et al., 2007).

Detección mediante fluorescencia de rayos $X$ de reflexión total. La tendencia en cuanto a la precisión y exactitud cuándo se realiza la detección por TXRF es similar a la observada por los otros métodos de detección empleados, aunque en general se observaron varianzas mayores respecto a la técnica de absorción atómica, como ha sido reportado anteriormente por Nečemer et al. (2008), la precisión está relacionada con la concentración de los analitos en las muestras de referencia y el límite de cuantificación (Montero et al., 2007; Martínez et al., 2008). Para la muestra de rango menor, $\mathrm{N}^{\circ} 6$ (Cuadro 7), se observaron varianzas altas para los elementos $\mathrm{Mg}, \mathrm{P}, \mathrm{Cu}$ y $\mathrm{Zn}$. El método resultó exacto y preciso en la determinación de Ca para las dos muestras de referencia que cubren los dos rangos. En la muestra $\mathrm{N}^{\circ} 11$ (Cuadro 8) donde el rango de concentración de $\mathrm{P}, \mathrm{Mg}, \mathrm{K}, \mathrm{Zn}$ y Cu es significativamente mayor se observa una disminución importante de la varianza. En general el método es más exacto y preciso en el rango representado por la muestra de referencia $\mathrm{N}^{\mathrm{o}} 11$ con la excepción de $\mathrm{Mg}$, para el cual no resultó exacta la técnica de detección.

\section{Evaluación del tiempo de análisis}

Se observó que para el método de microondas se toman 245 minutos para la digestión y análisis de 12 muestras, lo cual permiten hacer hasta 2 lotes de 12 muestras por día, para un total de 24 muestras/día lo que conlleva a lograr procesar hasta 96 muestras por semana (Cuadro 9).

Para la digestión seca se toman 2,230 minutos entre la digestión propiamente dicha y el análisis, aunque el tiempo es mayor, se digieren muchas más muestras que van de 40 a 60 muestras dependiendo del tamaño de la mufla y el de los crisoles, de esta forma se pueden digerir 60 muestras en 3 días, arrojando un promedio de 15 muestras/día.

Bajo las condiciones en las que se realizó esta investigación, el método de DAAM resultó mucho más rápido que el de DVS, pudiendo analizarse semanalmente por DAAM el doble de muestra que por DVS.

En cuanto a la detección se refiere, la determinación por separado de cada elemento por las distintas técnicas utilizadas comúnmente en el laboratorio (espectroscopia atómica y espectroscopia molecular) implica un tiempo de análisis significativamente mayor (50 muestras 
Cuadro 7. Concentración de $\mathrm{P}, \mathrm{K}, \mathrm{Ca}, \mathrm{Mg}, \mathrm{Zn}$ y $\mathrm{Cu}$ en la muestra vegetal certificada $\mathrm{N}^{\circ} 6$ (CNA-Chile) determinada por dos métodos de digestión y detección por TXRF

\begin{tabular}{|c|c|c|c|c|c|c|c|c|}
\hline Elemento & $\begin{array}{l}\text { VR } \\
(\%)\end{array}$ & $\begin{array}{l}\text { CV } \\
(\%)\end{array}$ & $\begin{array}{c}\text { DAAM } \\
(\%)\end{array}$ & $\begin{array}{c}\text { Er DAAM } \\
(\%)\end{array}$ & $\begin{array}{l}\text { CV } \\
(\%)\end{array}$ & $\begin{array}{c}\text { DVS } \\
(\%)\end{array}$ & $\begin{array}{c}\text { Er DVS } \\
(\%)\end{array}$ & $\begin{array}{l}\text { CV } \\
(\%)\end{array}$ \\
\hline $\mathrm{P}$ & $0.086 \mathrm{aA}$ & 10 & $0.0985 \mathrm{a}$ & 14.50 & 9 & $0.109 \mathrm{~A}$ & 27 & 11 \\
\hline K & $0.64 \mathrm{aA}$ & 8 & $0.75 \mathrm{~b}$ & 15.00 & 4 & $0.51 \mathrm{~B}$ & 20 & 6 \\
\hline $\mathrm{Ca}$ & $1.25 \mathrm{aA}$ & 7 & $1.30 \mathrm{a}$ & 4.00 & 7 & $1.21 \mathrm{~A}$ & 3 & 7 \\
\hline $\mathrm{Mg}$ & $0.175 \mathrm{aA}$ & 8 & $0.16 \mathrm{a}$ & 15.00 & 25 & $0.17 \mathrm{~A}$ & 3 & 23 \\
\hline $\mathrm{Cu}^{*}$ & $5.10 \mathrm{aA}$ & 22 & $11.00 \mathrm{a}$ & 78.00 & 48 & $6.60 \mathrm{~A}$ & 29 & 21 \\
\hline $\mathrm{Zn} *$ & $19.00 \mathrm{aA}$ & 10 & $39.00 \mathrm{~b}$ & 100.00 & 13 & $26.00 \mathrm{~A}$ & 37 & 25 \\
\hline
\end{tabular}

$\mathrm{VR}=$ valor real; $\mathrm{DAAM}=$ digestión ácida asistida por microondas; $\mathrm{DVS}=$ digestión vía seca; $\mathrm{Er}=$ error relativo porcentual. * Concentración en $\mathrm{mg} \mathrm{kg}^{-1}$.

Medias seguidas por la misma letra minúscula en la fila para VR y DAAM y mayúscula para VR y DVS no presentan diferencias estadísticas (Tukey, $\mathrm{p}<0.05$ ).

Cuadro 8. Concentración de $\mathrm{P}, \mathrm{K}, \mathrm{Ca}, \mathrm{Mg}, \mathrm{Zn}$ y $\mathrm{Cu}$ en la muestra vegetal certificada $\mathrm{N}^{\circ} 11$ (CNA-Chile) determinada por dos métodos de digestión y detección por TXRF

\begin{tabular}{|c|c|c|c|c|c|c|c|c|}
\hline Elemento & $\begin{array}{l}\text { VR } \\
(\%)\end{array}$ & $\begin{array}{l}\mathrm{CV} \\
(\%)\end{array}$ & $\begin{array}{c}\text { DAAM } \\
(\%)\end{array}$ & $\begin{array}{c}\text { Er DAAM } \\
(\%)\end{array}$ & $\begin{array}{l}\mathrm{CV} \\
(\%)\end{array}$ & $\begin{array}{c}\text { DVS } \\
(\%)\end{array}$ & $\begin{array}{c}\text { Er DVS } \\
(\%)\end{array}$ & $\begin{array}{l}\mathrm{CV} \\
(\%)\end{array}$ \\
\hline $\mathrm{P}$ & $0.229 \mathrm{aA}$ & 5 & $0.236 \mathrm{a}$ & 14 & 4 & $0.26 \mathrm{~A}$ & 17 & 10 \\
\hline K & $1.64 \mathrm{aA}$ & 5 & $1.70 \mathrm{a}$ & 4 & 6 & $1.50 \mathrm{~A}$ & 9 & 10 \\
\hline $\mathrm{Ca}$ & $2.07 \mathrm{aA}$ & 4 & $2.00 \mathrm{a}$ & 3 & 7 & $2.00 \mathrm{~A}$ & 3 & 6 \\
\hline $\mathrm{Mg}$ & $0.38 \mathrm{aA}$ & 5 & $0.26 \mathrm{~b}$ & 32 & 15 & $0.26 \mathrm{~B}$ & 32 & 8 \\
\hline $\mathrm{Cu}^{*}$ & $19.00 \mathrm{aA}$ & 11 & $18.00 \mathrm{a}$ & 5 & 17 & $19.00 \mathrm{~A}$ & 0 & 11 \\
\hline $\mathrm{Zn} *$ & $58.00 \mathrm{aA}$ & 3 & $65.00 \mathrm{a}$ & 12 & 5 & $66.00 \mathrm{~A}$ & 14 & 11 \\
\hline
\end{tabular}

$\mathrm{VR}=$ valor real; $\mathrm{DAAM}=$ digestión ácida asistida por microondas; $\mathrm{DVS}=$ digestión vía seca; $\mathrm{Er}=$ error relativo porcentual. * Concentración en $\mathrm{mg} \mathrm{kg}^{-1}$.

Medias seguidas por la misma letra minúscula en la fila para VR y DAAM y mayúscula para VR y DVS no presentan diferencias estadísticas (Tukey, $\mathrm{p}<0.05$ ).

Cuadro 9. Tiempo de realización de análisis por ambos métodos de digestión

\begin{tabular}{lrr}
\hline \multicolumn{1}{c}{ Actividad } & $\begin{array}{r}\text { DAAM } \\
\text { (minutos) }\end{array}$ & \multicolumn{1}{c}{ DVS } \\
\hline Preparación del material & 100 & 480 \\
Pesaje & $35-40$ & $80-90$ \\
Digestión & 35 & 540 \\
Enfriado & 40 & 120 \\
Aplicación de reactivos y reposo & 0 & 960 \\
Trasvasado & 30 & 40 \\
Total & 245 & 2230 \\
\hline
\end{tabular}

DAAM = digestión ácida asistida por microondas (12 muestras); DVS= digestión vía seca (40 muestras) 
requieren 6 horas hombre por cada elemento) comparado con TXRF en la cual no se preparan patrones normales de calibración, se puede realizar la determinación simultánea de todos los elementos en un tiempo de 15 minutos por muestra (50 muestras requieren $12.5 \mathrm{~h}$ hombre por el total de los elementos).

\section{Conclusiones}

L os métodos de DAAM y DVS permiten obtener resultados exactos y precisos en el análisis foliar de caña de azúcar para los elementos $\mathrm{K}$ y Ca cuando se utilizan las técnicas de espectroscopía de emisión y absorción atómica, o la técnica TXRF, especialmente en el rango de concentración mayor al 1\% p/p. En las muestras tomadas en campo se observa concordancia entre ambos métodos de digestión para los elementos $\mathrm{Ca}, \mathrm{Mg}$ y P. Para el K el método de DVS presenta valores menores respecto a DAAM. La determinación de $P$ vía TXRF muestra valores significativamente mayores, respecto a la técnica colorimétrica en las muestras de campo. En la determinación de $\mathrm{Zn}$ y $\mathrm{Cu}$ se presentan desviaciones y varianzas que están asociadas a las concentraciones bajas en las muestras y su relación con el blanco de reactivos, por lo que se requieren coeficientes de dilución menores, independientemente del método de detección. En general, la precisión y exactitud están relacionadas principalmente al rango de concentración de cada elemento en las muestras y el límite de cuantificación. No se encontraron diferencias apreciables en cuanto al desempeño analítico de ambos métodos de digestión, por tanto la DAAM, al permitir un mayor número de muestras procesadas por unidad de tiempo tiene ventajas importantes sobre la DVS. La detección por TXRF presenta menor eficiencia analítica en cuanto a exactitud y precisión en algunos elementos, debido a la dilución empleada ( $\mathrm{Zn}$ ), a interferencias espectrales en el caso del $\mathrm{P}$ o a la naturaleza ligera del elemento $(\mathrm{Mg})$. Sin embargo, dado que esta técnica permite la determinación simultánea de todos los analitos en el mismo spot de muestra, representa una alternativa interesante, que debería ser evaluada con menores diluciones de la muestra en el proceso de digestión, porque implica una reducción adicional del tiempo de análisis.

\section{Agradecimiento}

A 1 CDCHT-UCLA por el financiamiento de este trabajo a través del proyecto 014-AG-2012.

\section{Bibliografía}

APHA (American Public Health Association )-AWWA (American Water Works Association)-WPCF (Water Pollution Control Federation). 2005. Standard methods for the examination of water and wastewater. Ascorbic acid method 4500- P. E.21St. Ed., New York, p. 4-153.

Bocca, B., Aliminti, A., Forte, G., Petrucci, F., Pirola, C., Senofonte, O., Violante. N. 2003. Highthroughput microwave-digestion procedures to monitor neurotoxic elements in body fluids by means of inductively couple plasma mass Spectrometry. Anal. Bioanal. Chem., 377(1): 6570.

Bruker. 2011a. Spectra 7. Software for acquisition and evaluation of XRF spectra. User Manual. Bruker Nano GmbH, Berlin, Germany, 68 p.

Bruker. 2011b. S2 PicofoxTM. TXRF Spectrometer for element analysis. User Manual. Bruker Nano GmbH, Berlin, Germany, 118 p.

Dapiaggi, M., Leva, F., Rabuffetti, D., Ayrault, S., Gaudry, A., Cenci, R.M. 2001. Reliability and accuracy of environmental analytical data on moss samples: An interlaboratory comparison. Environ. Technol., 22(10): 1183-1192.

De La Calle, I., Cabaleiro, N., Romero, V., Lavilla, I., Vendicho, C. 2013. Sample pretreatment strategies for total reflection X-ray fluorescence analysis: A tutorial review. Spectrochimica Acta Part B: Atomic Spectroscopy 90: 23-54.

FAO (Food and Agriculture Organization of the United Nations). 2008. FAO fertilizer and plant nutrition bulletin: Guide to laboratory establishment for plant nutrient analysis. FAO, Rome, Italy, 203 p.

Hansen, T.H., Laursen, K.H., Persson, D.P., Pedas, P., Husted, S., Schjoerring, J.K. 2009. Micro-scaled high-throughput digestion of plant tissue samples for multi-elemental analysis. Plant Methods, 5(12) doi: 10.1186/1746-4811-5-12.

INFOSTAT. 2002. InfoStat versión 1.1. Grupo InfoStat. Fac. Cs. Agr. Universidad Nacional de Córdoba, Argentina.

Kalra, Y.P. 1998. Handbook of reference methods for plant analysis. Soil and Plant Analysis Council, Inc. NewYork. USA: CRC Press; p. 300.

Marguí, E. 2006. Analytical Methodologies based on X-ray fluorescence spectrometry (XRF) and inductively coupled plasma spectroscopy (ICP) for the assessment of metal dispersal around mining environments. Doctoral Thesis, University of Girona, Spain, p. 2, 3, 49, 50. 
Martínez, T., Lartigue, J., Zarazua, G., Avila-Pérez, P., Navarrete, M., y Tejeda, S. 2008. Application of the Total Reflection X-ray fluorescence technique to trace elements determination in tobacco. Spectrochimica Acta Part B: Atomic Spectroscopy 63(12): 1469-1472.

Montero, A., Estévez, J.R., y Padilla, R. 2007. Heavy metal analysis of rainwaters: a comparison of TXRF and ASV analytical capabilities. J. Radioanal Nucl. Ch., 273(2): 427-433.

Nečemer, M., Kump, P., Ščančar, J., Jaćimović, R., Simčič, J., Pelicon, P., Budnar, M., Jeran, Z., Pongrac, P., Regvar, M., Vogel-Mikuš, K. 2008. Application of X-ray fluorescence analytical techniques in phytoremediation and plant biology studies. Spectrochim. Acta, Parte B: Atomic Spectroscopy 63(11): 1240-1247.

Paslawski, P., Migaszewski, Z.M. 2006. The quality of element determinations in plant materials by instrumental methods. Polish J. Environ. Stud., 15(2a): 154-164.

Shirin, K., Naseem, S., Bashir, E., Imad, S., Shafio, S. 2008. A comparison of digestion methods for the estimation of elements in Dodonaea Viscosa: a native flora of Wadh, Balochistan, Pakistan. J. Chem. Soc. Pak., 30(1): 90-95.
Sun, D.H., Waters, J.K., y Mawhinney, T.P. 2000. Determination of thirteen common elements in food samples by inductively coupled plasma atomic emission spectrometry: comparison of five digestion methods. J. AOAC Int., 83(5): 12181224.

Untea, A., Criste, R.D., y Vladescu, L. 2012. Development and validation of a microwave digestión - FAAS Procedure for $\mathrm{Cu}, \mathrm{Mn}$ and $\mathrm{Zn}$ determination in liver. Rev. Chim. (Bucharest), 63(4): 341-346.

Wasiliewska, M., Goessler, W., Zischka, M., Maichin, B., Knapp, G. 2002. Efficiency of oxidation in wet digestion procedures and influence from the residual organic carbon content on selected techniques for determination of trace elements. J. Anal. At. Spectrom. 17(9): 1121-1125.

Zudowska, J. Biziuk, M. 2008. Methodological evaluation of method for dietary heavy metal intake. J. Food Sci., 73(2): 21-29. 